\title{
Intersecting Systems of Signed Sets
}

\author{
Peter Borg \\ Department of Pure Mathematics, The Open University, \\ Walton Hall, Milton Keynes MK7 6AA, United Kingdom \\ p.borg.02@cantab.net
}

Submitted: Jul 4, 2006; Accepted: May 24, 2007; Published: May 31, 2007

Mathematics Subject Classification: 05D05

\begin{abstract}
A family $\mathcal{F}$ of sets is said to be (strictly) $E K R$ if no non-trivial intersecting sub-family of $\mathcal{F}$ is (as large as) larger than some trivial intersecting sub-family of $\mathcal{F}$.

For a finite set $X:=\left\{x_{1}, \ldots, x_{|X|}\right\}$ and an integer $k \geq 2$, we define $\mathcal{S}_{X, k}$ to be the family of signed sets given by

$$
\mathcal{S}_{X, k}:=\left\{\left\{\left(x_{1}, a_{1}\right), \ldots,\left(x_{|X|}, a_{|X|}\right)\right\}: a_{i} \in[k], i=1, \ldots,|X|\right\} .
$$

For a family $\mathcal{F}$, we define $\mathcal{S}_{\mathcal{F}, k}:=\bigcup_{F \in \mathcal{F}} \mathcal{S}_{F, k}$.

We conjecture that for any $\mathcal{F}$ and $k \geq 2, \mathcal{S}_{\mathcal{F}, k}$ is EKR, and strictly so unless $k=2$ and $\mathcal{F}$ has a particular property.

A well-known result (stated by Meyer and proved in different ways by Deza and Frankl, Engel, Erdôs et al., and Bollobás and Leader) supports this conjecture for $\mathcal{F}=\left(\begin{array}{c}{[n]} \\ r\end{array}\right)$. The main theorem in this paper generalises this result by establishing the truth of the conjecture for families $\mathcal{F}$ that are compressed with respect to some $f^{*} \in \bigcup_{F \in \mathcal{F}} F$ (i.e. $f \in F \in \mathcal{F}, f^{*} \notin F \Rightarrow(F \backslash\{f\}) \cup\left\{f^{*}\right\} \in \mathcal{F}$ ). We also confirm the conjecture for families $\mathcal{F}$ that are uniform and EKR.
\end{abstract}

\section{Introduction}

We start with some standard notation for sets. $\mathbb{N}$ is the set of positive integers $\{1,2, \ldots\}$. For $m, n \in \mathbb{N}, m<n$, the set $\{m, m+1, \ldots, n\}$ is denoted by $[m, n]$, and if $m=1$ then we also write $[n]$. For a set $X$, the power set $\{A: A \subseteq X\}$ of $X$ is denoted by $2^{X}$, and the sub-family of $2^{X}$ given by $\{Y \subseteq X:|Y|=r\}$ is denoted by $\left(\begin{array}{l}X \\ r\end{array}\right)$. The Cartesian product $X \times Y$ of two sets $X$ and $Y$ is the set $\{(x, y): x \in X, y \in Y\}$.

Next, we recall some basic definitions for families $\mathcal{F}$ of sets. $\mathcal{F}$ is said to be intersecting if $F_{1} \cap F_{2} \neq \phi$ for any $F_{1}, F_{2} \in \mathcal{F}$. If $\bigcap_{F \in \mathcal{F}} F \neq \phi$ then $\mathcal{F}$ is said to be trivial, otherwise non-trivial. $\mathcal{F}$ is said to be $(r-)$ uniform if $(r=)\left|F_{1}\right|=\left|F_{2}\right|$ for any $F_{1}, F_{2} \in \mathcal{F}$. 
In the following, unless otherwise stated, we consider sets and families that are nonempty and finite.

Let $U(\mathcal{F}):=\bigcup_{F \in \mathcal{F}} F$. For any $f \in U(\mathcal{F})$, let

$$
\begin{gathered}
\mathcal{F}(f):=\{F \in \mathcal{F}: f \in F\}, \quad \mathcal{F}\langle f\rangle:=\{F \backslash\{f\}: F \in \mathcal{F}(f)\}, \\
\mathrm{O}_{\mathcal{F}}(f):=\left\{f^{\prime} \in U(\mathcal{F}): \mathcal{F}\left(f^{\prime}\right)=\mathcal{F}(f)\right\} .
\end{gathered}
$$

We call $\mathcal{F}(f)$ a star of $\mathcal{F}$ and $\mathrm{O}_{\mathcal{F}}(f)$ the $\mathcal{F}$-orbit of $f$. Let

$$
L(\mathcal{F}):=\{f \in U(\mathcal{F}): \mathcal{F}(f) \text { is a largest star of } \mathcal{F}\} .
$$

We denote the set of extremal (i.e. largest) intersecting sub-families of $\mathcal{F}$ by $\operatorname{ex}(\mathcal{F})$. $\mathcal{F}$ is said to have the Erdós-Ko-Rado (EKR) property if $\{\mathcal{F}(f): f \in L(\mathcal{F})\} \subseteq \operatorname{ex}(\mathcal{F})$, and to have the strict $E K R$ property if $\{\mathcal{F}(f): f \in L(\mathcal{F})\}=\operatorname{ex}(\mathcal{F})$; we abbreviate, saying that $\mathcal{F}$ is $E K R$ or strictly $E K R$, respectively.

A classical result in the literature on intersecting systems is the EKR Theorem [6], which says that if $r \leq n / 2$ then $\left(\begin{array}{c}{[n]} \\ r\end{array}\right)$ is EKR. Many other EKR-type results were proved after the publication of [6]; two good survey papers are [3] and [7].

We now define signed sets and give a brief review of EKR-type results that are most relevant to the results and conjecture concerning signed sets that we present in this paper. The 'signed sets' terminology was introduced in [2], but the presentation here is different and more general.

Let $X:=\left\{x_{1}, \ldots, x_{|X|}\right\}$. Let $y_{1}, \ldots, y_{|X|} \in \mathbb{N}$. We call the set $\left\{\left(x_{1}, y_{1}\right), \ldots,\left(x_{|X|}, y_{|X|}\right)\right\}$ a $k$-signed $|X|$-set if $\left|\left\{y_{1}, \ldots, y_{|X|}\right\}\right|=k$. For $k \geq 2$, we define $\mathcal{S}_{X, k}$ to be the family of $(\leq k)$-signed $|X|$-sets given by

$$
\mathcal{S}_{X, k}:=\left\{\left\{\left(x_{1}, a_{1}\right), \ldots,\left(x_{|X|}, a_{|X|}\right)\right\}: a_{i} \in[k], i=1, \ldots,|X|\right\} .
$$

We need to define $\mathcal{S}_{\phi, k}:=\phi$. For a family $\mathcal{F}$ of sets, we define

$$
\mathcal{S}_{\mathcal{F}, k}:=\bigcup_{F \in \mathcal{F}} \mathcal{S}_{F, k}
$$

The following is the basic result for signed sets.

Theorem 1.1 (Berge [1], Livingston [11]) (i) $\mathcal{S}_{[r], k}$ is EKR, and (ii) strictly so unless $k=2$.

Berge originally proved Theorem 1.1(i), and Livingston determined the extremal case. Other proofs of this result were given by Gronau [8] and Moon [13].

Holroyd and Talbot [9] recently showed that if $\mathcal{F}$ is an EKR family of independent $r$-sets of a graph then $\mathcal{S}_{\mathcal{F}, k}$ is EKR; however, their proof carries forward to the following generalisation of Theorem 1.1(i).

Theorem 1.2 (Holroyd, Talbot [9]) If $\mathcal{F}$ is $r$-uniform and EKR then $\mathcal{S}_{\mathcal{F}, k}$ is EKR. 
The next generalisation of Theorem 1.1 is a well-known result that was first stated by Meyer [12] and proved by Deza and Frankl [3].

Theorem 1.3 (Meyer [12], Deza and Frankl [3]) For $r \leq n$ and $k \geq 2$,

(i) $\mathcal{S}_{\left(\begin{array}{c}{[n]} \\ r\end{array}\right), k}$ is EKR, and

(ii) strictly so unless $r=n \geq 3$ and $k=2$.

Engel [4], Erdôs et al. [5], and Bollobás and Leader [2] gave other proofs of Theorem 1.3 based on variants of the circle method used by Katona [10] in his alternative proof of the EKR Theorem.

Note that Theorem 1.3(i) with $r \leq n / 2$ follows from Theorem 1.2 and the EKR Theorem. Also note that the case $r>n / 2$ in Theorem 1.3 provides an example of a family $\mathcal{F}$ such that $\mathcal{S}_{\mathcal{F}, k}$ is EKR but $\mathcal{F}$ is not.

This paper is motivated by the question "Do families $\mathcal{F}$ such that $\mathcal{S}_{\mathcal{F}, k}$ is not EKR for some $k \geq 2$ exist after all?". We conjecture that the answer is "no". Our main result provides a strong generalisation of Theorem 1.3 by establishing the truth of our conjecture for families $\mathcal{F}$ that are compressed wrt (with respect to) an element $f^{*} \in U(\mathcal{F})$, i.e. families $\mathcal{F}$ for which $f \in F \in \mathcal{F} \backslash \mathcal{F}\left(f^{*}\right)$ implies $(F \backslash\{f\}) \cup\left\{f^{*}\right\} \in \mathcal{F}\left(f^{*}\right)$. We are also concerned with strict and non-strict EKR cases.

Conjecture 1.4 Let $\mathcal{F}$ be any family of sets, and let $k \geq 2$. Then

(i) $\mathcal{S}_{\mathcal{F}, k}$ is $E K R$;

(ii) $\mathcal{S}_{\mathcal{F}, k}$ is strictly EKR unless $k=2$ and $\left|\mathrm{O}_{\mathcal{F}}(f)\right| \geq 3$ for some $(f, 1) \in L\left(\mathcal{S}_{\mathcal{F}, 2}\right)$.

Theorem 1.5 Conjecture 1.4 is true if $\mathcal{F} \subseteq 2^{[n]}$ is compressed wrt 1.

We also confirm Conjecture 1.4(ii) for families $\mathcal{F}$ as in Theorem 1.2.

Theorem 1.6 Conjecture 1.4 is true if $\mathcal{F}$ is $r$-uniform and EKR.

\section{An auxiliary result for the special case $k=2$}

For a signed set $A$ and integers $q$ and $k$, let

$$
\theta_{k}^{q}(A):=\{(x, a+q \text { modulo } k):(x, a) \in A\} .
$$

For $q=1$, we also write $\theta_{k}(A)$.

Clearly, if $k=2$ then $\theta_{2}(A)$ is the unique set in $\mathcal{S}_{X, 2}$ that does not intersect $A$. Thus, for $\mathcal{A} \subset \mathcal{S}_{X, 2}$,

$$
\mathcal{A} \in \operatorname{ex}\left(\mathcal{S}_{X, 2}\right) \Leftrightarrow\left|\mathcal{A} \cap\left\{A, \theta_{2}(A)\right\}\right|=1 \text { for all } A \in \mathcal{S}_{X, 2} .
$$

Note that stars of $\mathcal{S}_{X, 2}$ are members of $\operatorname{ex}\left(\mathcal{S}_{X, 2}\right)$, and that not all members of ex $\left(\mathcal{S}_{X, 2}\right)$ are stars unless $|X| \leq 2$.

We generalise (1) in the following direction. 
Theorem 2.1 Let $I \subseteq \mathbb{N}$. For each $i \in I$, let $X_{i}$ be a finite set and let $\mathcal{A}_{i} \subset \mathcal{S}_{X_{i}, 2}$. Suppose $\bigcup_{i \in I} \mathcal{A}_{i}$ is intersecting. Then $\mathcal{A}_{i} \in \operatorname{ex}\left(\mathcal{S}_{X_{i}, 2}\right)$ holds for all $i \in I$ iff $X:=\bigcap_{i \in I} X_{i} \neq$ $\phi$ and, for some $\mathcal{X} \in \operatorname{ex}\left(\mathcal{S}_{X, 2}\right)$ and for all $i \in I, \mathcal{A}_{i}=\left\{A \in \mathcal{S}_{X_{i}, 2}: A \cap(X \times 2) \in \mathcal{X}\right\}$.

Proof. The sufficiency condition is straightforward since then, for all $i \in I, \mathcal{A}_{i}$ satisfies (1). Now assume that for all $i \in I, \mathcal{A}_{i} \in \operatorname{ex}\left(\mathcal{S}_{X_{i}, 2}\right)$. We prove the necessary condition by induction on $m:=\min \left\{\left|X_{i}\right|: i \in I\right\}$.

Suppose there exist $i_{1}, i_{2}, l \in I$ such that $A_{i_{1}} \cap A_{i_{2}} \cap\left(X_{l} \times[2]\right)=\phi$ for some $A_{i_{1}} \in \mathcal{A}_{i_{1}}$ and $A_{i_{2}} \in \mathcal{A}_{i_{2}}$. Let $B_{p}:=A_{i_{p}} \cap\left(X_{l} \times[2]\right), p=1,2$. Let $Y:=X_{l} \backslash\left(X_{i_{1}} \cup X_{i_{2}}\right)$. If $Y \neq \phi$ then choose $C \in \mathcal{S}_{Y, 2}$, otherwise take $C:=\phi$. Let $D:=B_{1} \backslash\left(X_{i_{2}} \times[2]\right)$ and $E:=B_{2} \cup \theta_{2}(D) \cup C \in \mathcal{S}_{X_{l}, 2}$. So $E \cap A_{i_{1}}=\phi$ and $\theta_{2}(E) \cap A_{i_{2}}=\phi$. Therefore $E, \theta_{2}(E) \notin \mathcal{A}_{l}$, and hence, by (1), we have $\mathcal{A}_{l} \notin \operatorname{ex}\left(\mathcal{S}_{X_{l}, 2}\right)$, a contradiction. Thus,

$$
\text { for any } A, B \in \bigcup_{i \in I} \mathcal{A}_{i} \text { and } l \in I, A \cap B \cap\left(X_{l} \times[2]\right) \neq \phi \text {. }
$$

Let $\mathcal{X}:=\left\{A \cap(X \times 2): A \in \bigcup_{i \in I} \mathcal{A}_{i}\right\}\left(X:=\bigcap_{i \in I} X_{i}\right)$. Let $j \in I$ such that $\left|X_{j}\right|=m$. Suppose $X=X_{j}$. So $\mathcal{X} \supseteq \mathcal{A}_{j}$ and, by (2) (with $l=j$ ), $\mathcal{X}$ is intersecting. Since $\mathcal{A}_{j} \in \operatorname{ex}\left(\mathcal{S}_{X_{j}, 2}\right)$, it follows that $\mathcal{X}=\mathcal{A}_{j}$, and hence result. It will be clear from the following that we have also just covered the basis of induction $m=1$.

Now suppose $X \neq X_{j}$. So there exists $h \in I$ such that $X_{j} \backslash X_{h} \neq \phi$. Let $x_{j} \in X_{j} \backslash X_{h}$. Let $X_{j}^{\prime}:=X_{j} \backslash\left\{x_{j}\right\}$, and for each $i \in I \backslash\{j\}$, let $X_{i}^{\prime}:=X_{i}$. So $\bigcap_{i \in I} X_{i}^{\prime}=X$. If $X_{j}^{\prime}=\phi$ then $X_{j} \cap X_{h}=\phi$, and hence $\mathcal{A}_{j}=\phi$ or $\mathcal{A}_{h}=\phi$, a contradiction. Therefore $m^{\prime}:=$ $\min \left\{\left|X_{i}^{\prime}\right|: i \in I\right\}=\left|X_{j}^{\prime}\right|=m-1 \geq 1$. For each $i \in I \backslash\{j\}$, we have $\mathcal{A}_{i}^{\prime}:=\mathcal{A}_{i} \in \operatorname{ex}\left(\mathcal{S}_{X_{i}^{\prime}, 2}\right)$. Let $\mathcal{A}_{j}^{\prime}:=\left\{A \backslash\left\{\left(x_{j}, 1\right),\left(x_{j}, 2\right)\right\}: A \in \mathcal{A}_{j}\right\} \subseteq \mathcal{S}_{X_{j}^{\prime}, 2}$. By (2), for any $A, B \in \bigcup_{i \in I} \mathcal{A}_{i}$, $A \cap B \cap\left(X_{h} \times[2]\right) \neq \phi$; hence $\bigcup_{i \in I} \mathcal{A}_{i}^{\prime}$ is intersecting. Suppose $\mathcal{A}_{j}^{\prime} \notin \operatorname{ex}\left(\mathcal{S}_{X_{j}^{\prime}, 2}\right)$. Then, by (1), $A_{j}^{\prime}, \theta_{2}\left(A_{j}^{\prime}\right) \notin \mathcal{A}_{j}^{\prime}$ for some $A_{j}^{\prime} \in \mathcal{S}_{X_{j}^{\prime}, 2}$. So $A_{j}:=A_{j}^{\prime} \cup\left\{x_{j}, 1\right\} \notin \mathcal{A}_{j}$ and $\theta_{2}\left(A_{j}\right) \notin \mathcal{A}_{j}$, which, by (1), contradicts $\mathcal{A}_{j} \notin \operatorname{ex}\left(\mathcal{S}_{X_{j}, 2}\right)$. Hence $\mathcal{A}_{j}^{\prime} \in \operatorname{ex}\left(\mathcal{S}_{X_{j}^{\prime}, 2}\right)$. Clearly, the result follows immediately after applying the inductive hypothesis for the families $\mathcal{A}_{i}^{\prime}, i \in I$.

Corollary 2.2 If $f \in U(\mathcal{F})$ and $\mathcal{A} \in \operatorname{ex}\left(\mathcal{S}_{\mathcal{F}(f), 2}\right)$ then:

(i) $\mathcal{A}=\left\{F \in \mathcal{F}: F \cap\left(\mathrm{O}_{\mathcal{F}(f)}(f) \times[2]\right) \in \mathcal{X}\right\}$ for some $\mathcal{X} \in \operatorname{ex}\left(\mathcal{S}_{\mathrm{O}_{\mathcal{F}(f)}(f), 2}\right)$;

(ii) if $\left|\mathrm{O}_{\mathcal{F}(f)}(f)\right| \leq 2$ then $\mathcal{A}=\mathcal{S}_{\mathcal{F}, 2}\left(\left(f^{\prime}, b\right)\right)$ for some $\left(f^{\prime}, b\right) \in \mathrm{O}_{\mathcal{F}(f)}(f) \times[2]$.

Proof. Let $\mathcal{B}:=\mathcal{S}_{\mathcal{F}, 2}((f, 1))$. Set $n:=|\mathcal{F}(f)|$, and let $X_{1}, \ldots, X_{n}$ be the sets in $\mathcal{F}(f)$. For each $i \in[n]$, let $\mathcal{A}_{i}:=\mathcal{A} \cap \mathcal{S}_{X_{i}, 2}, \mathcal{B}_{i}:=\mathcal{B} \cap \mathcal{S}_{X_{i}, 2}$. So $\bigcup_{i=1}^{n} \mathcal{A}_{i}$ and $\bigcup_{i=1}^{n} \mathcal{B}_{i}$ are partitions of $\mathcal{A}$ and $\mathcal{B}$ respectively. By $(1), \mathcal{B}_{i} \in \operatorname{ex}\left(\mathcal{S}_{X_{i}, 2}\right), i=1, \ldots, n$, and hence $\mathcal{B} \in \operatorname{ex}\left(\mathcal{S}_{\mathcal{F}(f), 2}\right)$. Thus, if $\mathcal{A}_{i} \notin \operatorname{ex}\left(\mathcal{S}_{X_{i}, 2}\right)$ for some $i \in[n]$ then $|\mathcal{A}|<|\mathcal{B}|$, but this contradicts $\mathcal{A} \in \operatorname{ex}\left(\mathcal{S}_{\mathcal{F}(f), 2}\right)$. So $\mathcal{A}_{i} \in \operatorname{ex}\left(\mathcal{S}_{X_{i}, 2}\right), i=1, \ldots, n$. By Theorem 2.1, for some $\mathcal{X} \in \operatorname{ex}\left(\mathcal{S}_{X, 2}\right)$ and for all $\left.i \in[n], \mathcal{A}_{i}=\left\{A \in \mathcal{S}_{X_{i}, 2}: A \cap(X \times 2) \in \mathcal{X}\right)\right\}$, where $X=\bigcap_{i=1}^{n} X_{i} \ni f$. So (i) follows if $X=\mathrm{O}_{\mathcal{F}(f)}(f)$. Let $x \in U(\mathcal{F}(f))$. If $x \notin X$ then there exists $j \in[n]$ such that $x \notin X_{j}$, and hence $x \notin \mathrm{O}_{\mathcal{F}(f)}(f)$; contrapositively, if $x \in \mathrm{O}_{\mathcal{F}(f)}(f)$ then $x \in X$. So $\mathrm{O}_{\mathcal{F}(f)}(f) \subseteq X$. If $x \in X$ then $\mathcal{F}(f)(x)=\mathcal{F}(f)$. So $X \subseteq \mathrm{O}_{\mathcal{F}(f)}(f)$, and hence $X=\mathrm{O}_{\mathcal{F}(f)}(f)$ indeed.

Suppose $\left|\mathrm{O}_{\mathcal{F}(f)}(f)\right| \leq 2$. So $1 \leq\left|\mathrm{O}_{\mathcal{F}(f)}(f)\right|=|X| \leq 2$, and it is trivial that $\mathcal{X}$ can only be a star in this case. Hence (ii). 
The strict and non-strict EKR cases for $k=2$ in each of Theorems 1.5 and 1.6 will be determined using Theorem 2.1, Corollary 2.2 and the following fact.

Proposition 2.3 If $\left|\mathrm{O}_{\mathcal{F}}(f)\right| \geq 3$ for some $(f, 1) \in L\left(\mathcal{S}_{\mathcal{F}, 2}\right)$ then $\mathcal{S}_{\mathcal{F}, 2}$ is not strictly EKR.

Proof. We have $\mathcal{F}\left(f_{1}\right)=\mathcal{F}\left(f_{2}\right)=\mathcal{F}\left(f_{3}\right)=\mathcal{F}(f)$ for some distinct $f_{1}, f_{2}, f_{3} \in \mathrm{O}_{\mathcal{F}}(f)$ (possibly, $f \in\left\{f_{1}, f_{2}, f_{3}\right\}$ ). It follows that for all $F \in \mathcal{F}(f), f_{1}, f_{2}, f_{3} \in F$. Define $Y_{1}:=\left\{\left(f_{1}, 1\right),\left(f_{2}, 1\right),\left(f_{3}, 1\right)\right\}, Y_{2}:=\left\{\left(f_{1}, 1\right),\left(f_{2}, 1\right),\left(f_{3}, 2\right)\right\}, Y_{3}:=\left\{\left(f_{1}, 1\right),\left(f_{2}, 2\right),\left(f_{3}, 1\right)\right\}$, $Y_{4}:=\left\{\left(f_{1}, 2\right),\left(f_{2}, 1\right),\left(f_{3}, 1\right)\right\}$. Clearly, the family $\bigcup_{F \in \mathcal{F}}\left\{Y_{i} \cup Z: i \in[4], Z \in \mathcal{S}_{F \backslash\left\{f_{1}, f_{2}, f_{3}\right\}, 2}\right\}$ is non-trivial, intersecting, and as large as $\mathcal{S}_{\mathcal{F}, 2}((f, 1))$, a largest star of $\mathcal{S}_{\mathcal{F}, 2}$.

\section{Proof of main result}

This section is dedicated to the proof of Theorem 1.5, which is based on two different compression - also known as shifting or pushing-up - methods. We refer the reader to [7] for a survey on compression applications in extremal set theory.

The first compression operation was used in [3] for the proof of Theorem 1.3. For $(a, b) \in[n] \times[2, k]$, let $\Delta_{a, b}: 2^{\mathcal{S}_{2}[n], k} \rightarrow 2^{\mathcal{S}_{2}[n], k}$ be defined by

$$
\Delta_{a, b}(\mathcal{A}):=\left\{\delta_{a, b}(A): A \in \mathcal{A}\right\} \cup\left\{A \in \mathcal{A}: \delta_{a, b}(A) \in \mathcal{A}\right\},
$$

where $\delta_{a, b}: \mathcal{S}_{2^{[n], k}} \rightarrow \mathcal{S}_{2^{[n]}, k}$ is defined by

$$
\delta_{a, b}(A):= \begin{cases}A \backslash\{(a, b)\} \cup\{(a, 1)\} & \text { if }(a, b) \in A \\ A & \text { otherwise }\end{cases}
$$

We say $\mathcal{A} \subseteq \mathcal{S}_{2^{[n]}, k}$ is $\Delta$-compressed if for all $(a, b) \in[n] \times[2, k], \Delta_{a, b}(\mathcal{A})=\mathcal{A}$.

The following lemma outlines the well-known fundamental properties of the compression $\Delta_{a, b}$, which are not difficult to prove.

Lemma 3.1 Let $\mathcal{A} \subseteq \mathcal{S}_{2^{[n]}, k}$. Let $(a, b) \in[n] \times[2, k]$. Then:

(i) $\left|\Delta_{a, b}(\mathcal{A})\right|=|\mathcal{A}|$;

(ii) if $\mathcal{A}$ is intersecting then $\Delta_{a, b}(\mathcal{A})$ is intersecting;

(iii) if $\mathcal{A}$ is intersecting and $\Delta$-compressed then $A_{1} \cap A_{2} \cap([n] \times[1]) \neq \phi$ for any $A_{1}, A_{2} \in \mathcal{A}$.

We next introduce our second compression operation. We take $K:=\{(1, b): b \in$ $[k]\} \cup\{(n, 1)\}$ and define $\lambda: \mathcal{S}_{2^{[n], k}} \rightarrow \mathcal{S}_{2^{[n]}, k}$ by

$$
\lambda(A):= \begin{cases}A \backslash\{(n, 1)\} \cup\{(1,1)\} & \text { if } A \cap K=\{(n, 1)\} ; \\ A \backslash\{(1, b),(n, 1)\} \cup\{(1,1),(n, b)\} & \text { if } A \cap K=\{(1, b),(n, 1)\} \\ A & \text { otherwise. }\end{cases}
$$

Similarly to $\Delta_{a, b}$, we define $\Lambda: 2^{\mathcal{S}_{2[n], k}} \rightarrow 2^{\mathcal{S}_{2}[n], k}$ by

$$
\Lambda(\mathcal{A}):=\{\lambda(A): A \in \mathcal{A}\} \cup\{A \in \mathcal{A}: \lambda(A) \in \mathcal{A}\} .
$$


At this point, we need to introduce some further notation. For $A \in \mathcal{S}_{F, k}$, let

$$
\gamma(A):=F
$$

For $\mathcal{A} \subseteq \mathcal{S}_{\mathcal{F}, k}$, let $\Gamma(\mathcal{A})$ be the sub-family of $\mathcal{F}$ given by

$$
\Gamma(\mathcal{A}):=\{\gamma(A): A \in \mathcal{A}\}=\left\{F \in \mathcal{F}: \mathcal{A} \cap \mathcal{S}_{F, k} \neq \phi\right\}
$$

Lemma 3.2 Let $\mathcal{F} \subseteq 2^{[n]}$ be compressed wrt 1 . Let $\mathcal{A} \subset \mathcal{S}_{\mathcal{F}, k}$ be intersecting and $\Delta$ compressed. Then:

(i) $|\Lambda(\mathcal{A})|=|\mathcal{A}|$;

(ii) $\Lambda(\mathcal{A}) \subset \mathcal{S}_{\mathcal{F}, k}$;

(iii) $B_{1} \cap B_{2} \cap([n] \times[1]) \neq \phi$ for any $B_{1}, B_{2} \in \Lambda(\mathcal{A})$.

Proof. (i) is straightforward.

If $A \in \mathcal{S}_{\mathcal{F}, k}$ and $\gamma(\lambda(A)) \neq \gamma(A)$ then $n \in \gamma(A), 1 \notin \gamma(A)$ and $\gamma(\lambda(A))=(\gamma(A) \backslash\{n\}) \cup$ $\{1\}$. Since $\mathcal{F}$ is compressed wrt 1 , it follows that $\Gamma(\Lambda(\mathcal{A})) \subseteq \mathcal{F}$. Hence (ii).

By Lemma 3.1, $A \cap A^{\prime} \cap([n] \times[1]) \neq \phi$ for any $A, A^{\prime} \in \mathcal{A}$. Let $B_{1}, B_{2} \in \Lambda(\mathcal{A})$. Then, for each $p \in[2], B_{p}=A_{p}$ or $B_{p}=\lambda\left(A_{p}\right)$ for some $A_{p} \in \mathcal{A}$. It is straightforward that (iii) holds if $B_{p}=A_{p}, p=1,2$, or $B_{p}=\lambda\left(A_{p}\right), p=1,2$. Without loss of generality, suppose $B_{1}=A_{1}, B_{2}=\lambda\left(A_{2}\right) \neq A_{2}$ and $B_{1} \cap B_{2} \cap([n] \times[1])=\phi$. It follows that $A_{1} \cap A_{2} \cap([n] \times[1])=\{(n, 1)\}$ and $A_{1} \neq \lambda\left(A_{1}\right) \in \mathcal{A}$. But then $\lambda\left(A_{1}\right) \cap A_{2} \cap([n] \times[1])=\phi$, a contradiction. Hence (iii).

Theorem 3.3 Let $1 \in J \subseteq[n]$. Let $\mathcal{F} \subseteq 2^{[n]}$ such that $\mathcal{F}$ is compressed wrt $j \in[n]$ iff $j \in J$. Let $\mathcal{A}^{*} \subset \mathcal{S}_{\mathcal{F}, k}$ be intersecting. Then

(i) $\left|\mathcal{A}^{*}\right| \leq\left|\mathcal{S}_{\mathcal{F}, k}((1,1))\right|$, and

(ii) equality holds iff $\mathcal{A}^{*}=\mathcal{S}_{\mathcal{F}, k}((j, b)),(j, b) \in J \times[k]$, or $k=2,\left|\mathrm{O}_{\mathcal{F}}(1)\right|>1$ and $\mathcal{A}^{*}=\left\{F \in \mathcal{F}: F \cap\left(\mathrm{O}_{\mathcal{F}}(1) \times[2]\right) \in \mathcal{X}\right\}, \mathcal{X} \in \operatorname{ex}\left(\mathcal{S}_{\mathrm{O}_{\mathcal{F}}(1), 2}\right)$.

Proof of Theorem 3.3(i). The case $n=2$ is trivial, so we assume $n>2$. Let $\mathcal{A}^{\prime}$ be a $\Delta$-compressed family resulting from $\mathcal{A}^{*}$ through repeated application, if necessary, of compressions $\Delta_{a, b}$. Let $\mathcal{A}:=\Lambda\left(\mathcal{A}^{\prime}\right)$. By Lemmas 3.1 and $3.2, \mathcal{A} \subset \mathcal{S}_{\mathcal{F}, k},|\mathcal{A}|=\left|\mathcal{A}^{*}\right|$ and

$$
\text { for any } A, B \in \mathcal{A}, A \cap B \cap Z \neq \phi \text {, }
$$

where $Z:=[n] \times[1]$.

Let $\mathcal{B}:=\{A \in \mathcal{A}((n, 1))((1,1)): A \cap B \cap Z=\{(n, 1)\}$ for some $B \in \mathcal{A}((n, 1))\}$. Let $\mathcal{A}_{1}:=\mathcal{A}((n, 1)) \backslash \mathcal{B}$. For $l \in[2, k]$, let $\mathcal{B}_{l}:=\{(A \backslash\{(n, 1)\}) \cup\{n, l\}: A \in \mathcal{B}\}$ and $\mathcal{A}_{l}:=$ $\mathcal{A}((n, l)) \cup \mathcal{B}_{l}$. If $\mathcal{A}((n, l)) \cap \mathcal{B}_{l} \neq \phi$ and $A \in \mathcal{A}((n, l)) \cap \mathcal{B}_{l}$ then $\delta_{n, l}(A) \cap B \cap Z=\{(n, 1)\}$ for some $B \in \mathcal{A}((n, 1))$, and hence $A \cap B \cap Z=\phi$, a contradiction to $(3)$. So $\mathcal{A}((n, l)) \cap \mathcal{B}_{l}=\phi$. Therefore

$$
\sum_{i=1}^{k}|\mathcal{A}((n, i))| \leq(|\mathcal{A}((n, 1))|-|\mathcal{B}|)+\sum_{l=2}^{k}(|\mathcal{A}((n, l))|+|\mathcal{B}|)=\sum_{i=1}^{k}\left|\mathcal{A}_{i}\right|
$$


Let $Z^{\prime}:=[n-1] \times[1]$. Suppose that for some $i \in[k]$ and $A, B \in \mathcal{A}_{i}, A \cap B \cap Z^{\prime}=\phi$. It is immediate by (3) that $i \notin[2, k]$. So $A, B \in \mathcal{A}_{1}$ and $A \cap B=\{(n, 1)\}$. By definition of $\mathcal{A}_{1},(1,1) \notin A \cup B$. But $\lambda(A) \in \mathcal{A}$ and $\lambda(A) \cap B \cap Z=\phi$, a contradiction to (3). Thus,

$$
\text { for any } i \in[k] \text { and } A, B \in \mathcal{A}_{i}, A \cap B \cap Z^{\prime} \neq \phi \text {. }
$$

Let $\mathcal{F}_{0}:=\mathcal{F} \backslash \mathcal{F}(n)$ and $\mathcal{F}_{1}:=\mathcal{F}\langle n\rangle$. Clearly, $\mathcal{F}_{0}$ and $\mathcal{F}_{1}$ are compressed wrt 1. Let $\mathcal{A}_{0}:=\mathcal{A} \cap \mathcal{S}_{\mathcal{F}_{0}, k}$. By (3), $\mathcal{A}_{0}$ is an intersecting sub-family of $\mathcal{S}_{\mathcal{F}_{0}, k}$. By $(5), \mathcal{A}_{i}\langle(n, i)\rangle$ is an intersecting sub-family of $\mathcal{S}_{\mathcal{F}_{1}, k}, i=1, \ldots, k$. The result now follows by induction on $n$ since

$$
\begin{aligned}
\left|\mathcal{A}^{*}\right| & =|\mathcal{A}|=\left|\mathcal{A}_{0}\right|+\sum_{i=1}^{k}|\mathcal{A}((n, i))| \leq\left|\mathcal{A}_{0}\right|+\sum_{i=1}^{k}\left|\mathcal{A}_{i}\langle(n, i)\rangle\right| \\
& \leq\left|\mathcal{S}_{\mathcal{F}_{0}, k}((1,1))\right|+k\left|\mathcal{S}_{\mathcal{F}_{1}, k}((1,1))\right|=\left|\mathcal{S}_{\mathcal{F}, k}((1,1))\right|,
\end{aligned}
$$

where the first inequality is obtained from (4).

We need to do more work to prove the extremal structures given in Theorem 3.3(ii). We start with a simple lemma that we will use often.

Lemma 3.4 Let $\mathcal{A} \subseteq \mathcal{S}_{\mathcal{F}, k}$ be intersecting. Suppose that for some $(a, b) \in U(\mathcal{F})$ and $F \in \mathcal{F}, \mathcal{S}_{F, k}((a, b)) \subseteq \mathcal{A}$. Then $\mathcal{A} \subseteq \mathcal{S}_{\mathcal{F}, k}((a, b))$.

Proof. It suffices to show that if $B \in \mathcal{S}_{\mathcal{F}, k} \backslash \mathcal{S}_{\mathcal{F}, k}((a, b))$ then $A \cap B=\phi$ for some $A \in \mathcal{S}_{F, k}((a, b))$. Let $C \in \mathcal{S}_{F, k} \backslash \mathcal{S}_{F, k}((a, b))$ such that $B \cap(F \times[k]) \subseteq C$. Clearly, for some $q \in[k-1], \theta_{k}^{q}(C) \in \mathcal{S}_{F, k}((a, b))$ and $B \cap \theta_{k}^{q}(C)=\phi$.

Lemma 3.5 Let $\mathcal{F} \subseteq 2^{[n]}$. Let $\mathcal{A} \subset \mathcal{S}_{\mathcal{F}, k}$ be intersecting, where $k \geq 3$. If $\mathcal{A} \neq \Delta_{a, b}(\mathcal{A})=$ $\mathcal{S}_{\mathcal{F}, k}\left(\left(a^{\prime}, b^{\prime}\right)\right)$ then $\mathcal{A}=\mathcal{S}_{\mathcal{F}, k}((a, b))$.

Proof. Since $\mathcal{A} \neq \Delta_{a, b}(\mathcal{A})=\mathcal{S}_{\mathcal{F}, k}\left(\left(a^{\prime}, b^{\prime}\right)\right)$, there exists $A \in \mathcal{A}$ such that $A \notin \mathcal{S}_{\mathcal{F}, k}\left(\left(a^{\prime}, b^{\prime}\right)\right)$ and $\delta_{a, b}(A) \in \mathcal{S}_{\mathcal{F}, k}\left(\left(a^{\prime}, b^{\prime}\right)\right)$. This implies that $\left(a^{\prime}, b^{\prime}\right)=(a, 1)$. Let $F:=\gamma(A)$. Let $\mathcal{A}_{F}:=\mathcal{A} \cap \mathcal{S}_{F, k}$. Clearly, $\Delta_{a, b}\left(\mathcal{A}_{F}\right)=\Delta_{a, b}(\mathcal{A}) \cap \mathcal{S}_{F, k}=\mathcal{S}_{F, k}((a, 1))$ and $\left|\mathcal{A}_{F}\right|=\left|\Delta_{a, b}\left(\mathcal{A}_{F}\right)\right|=$ $\left|\mathcal{S}_{F, k}((a, 1))\right|$. Thus, by Theorem 1.1, $\mathcal{A}_{F}=\mathcal{S}_{F, k}((c, d))$ for some $(c, d) \in F \times[k]$. Since $A \in \mathcal{A}_{F} \backslash \mathcal{S}_{F, k}((a, 1))$ and $\Delta_{a, b}\left(\mathcal{A}_{F}\right)=\mathcal{S}_{F, k}((a, 1))$, it follows that $(c, d)=(a, b)$. By Lemma 3.4, $\mathcal{A} \subseteq \mathcal{S}_{\mathcal{F}, k}((a, b))$. Since $|\mathcal{A}|=\left|\Delta_{a, b}(\mathcal{A})\right|=\left|\mathcal{S}_{\mathcal{F}, k}((a, b))\right|, \mathcal{A}=\mathcal{S}_{\mathcal{F}, k}((a, b))$.

For $F \in \mathcal{F}$ and $f, g \in U(\mathcal{F})$, let $\psi_{f, g}(F):=(F \backslash\{g\}) \cup\{f\}$.

Proposition 3.6 Let $\mathcal{F}$ and $J$ be as in Theorem 3.3. Then $L\left(\mathcal{S}_{\mathcal{F}, k}\right)=J \times[k]$.

Proof. Let $j \in J$ and $h \in[n]$. Then, since $\mathcal{F}$ is compressed wrt $j$,

$$
\begin{aligned}
& \left|\mathcal{S}_{\mathcal{F}, k}((h, b))\right|=\sum_{H \in \mathcal{F}(h)(j)}\left|\mathcal{S}_{H, k}((h, b))\right|+\sum_{G \in \mathcal{F}(h) \backslash \mathcal{F}(j)}\left|\mathcal{S}_{G, k}((h, b))\right| \\
= & \sum_{H \in \mathcal{F}(j)(h)}\left|\mathcal{S}_{H, k}((j, b))\right|+\sum_{G \in \mathcal{F}(h) \backslash \mathcal{F}(j)}\left|\mathcal{S}_{\psi_{j, h}(G), k}((j, b))\right| \leq\left|\mathcal{S}_{\mathcal{F}, k}((j, b))\right|
\end{aligned}
$$


with equality iff for all $F \in \mathcal{F}(j) \backslash \mathcal{F}(h), \psi_{h, j}(F) \in \mathcal{F}(h)$. This already gives $J \times[k] \subseteq L(\mathcal{F})$. Now consider equality in $(7)$. Suppose $h \notin J$. So there exist $F \in \mathcal{F} \backslash \mathcal{F}(h)$ and $f \in F$ such that $F_{1}:=\psi_{h, f}(F) \notin \mathcal{F}(h)$. Therefore $f \neq j$. If $j \notin F$ then $F_{2}:=\psi_{j, f}(F) \in \mathcal{F}(j) \backslash \mathcal{F}(h)$, $\psi_{h, j}\left(F_{2}\right)=F_{1}$, and hence $\psi_{h, j}\left(F_{2}\right) \notin \mathcal{F}(h)$; but this is a contradiction as it yields a strict inequality in (7). So $F \in \mathcal{F}(j) \backslash \mathcal{F}(h)$, and hence $F_{3}:=\psi_{h, j}(F) \in \mathcal{F}(h) \backslash \mathcal{F}(j)$. Thus $F_{4}:=\psi_{j, f}\left(F_{3}\right) \in \mathcal{F}(h)(j)$. Now $\psi_{h, f}(F)=\psi_{j, f}\left(\psi_{h, j}(F)\right)$, and hence $F_{1}=F_{4}$; but this contradicts $F_{4} \in \mathcal{F}(h)$ and $F_{1} \notin \mathcal{F}(h)$. So $h \in J$. Therefore $L(\mathcal{F}) \subseteq J \times[k]$. This establishes the result since we previously obtained $J \times[k] \subseteq L(\mathcal{F})$.

Lemma 3.7 Let $\mathcal{A}^{\prime}$ be as in the Proof of Theorem 3.3(i). Suppose $\mathcal{A}^{\prime} \neq \Lambda\left(\mathcal{A}^{\prime}\right)=$ $\mathcal{S}_{\mathcal{F}, k}((a, 1))$. Then $n \in J$ and $\mathcal{A}^{\prime}=\mathcal{S}_{\mathcal{F}, k}((n, 1))$.

Proof. Since $\mathcal{A}^{\prime} \neq \Lambda\left(\mathcal{A}^{\prime}\right)=\mathcal{S}_{\mathcal{F}, k}((a, 1))$, there exists $A^{\prime} \in \mathcal{A}^{\prime}$ such that $A^{\prime} \notin \mathcal{S}_{\mathcal{F}, k}((a, 1))$ and $\lambda\left(A^{\prime}\right) \in \mathcal{S}_{\mathcal{F}, k}((a, 1))$. So $a=1$ and $\gamma\left(A^{\prime}\right) \cap\{1, n\}=\{n\}$. Let $A:=\{(n, 1)\} \cup$ $\left\{(c, 2): c \in \gamma\left(A^{\prime}\right) \backslash\{n\}\right\}$. Since $\Lambda\left(\mathcal{A}^{\prime}\right)=\mathcal{S}_{\mathcal{F}, k}((1,1))$, exactly one of $A$ and $\lambda(A)(\neq A)$ is in $\mathcal{A}^{\prime}$. Recall that we arrived at (3) using the fact that, by Lemma 3.1, $A_{1} \cap A_{2} \cap([n] \times[1]) \neq \phi$ for any $A_{1}, A_{2} \in \mathcal{A}^{\prime}$. Since $\lambda(A) \cap A^{\prime} \cap([n] \times[1])=\phi$, it follows that $A \in \mathcal{A}^{\prime}$, and hence, since $A \cap([n] \times[1])=\{(n, 1)\}, \mathcal{A}^{\prime} \subseteq \mathcal{S}_{\mathcal{F}, k}((n, 1))$. Since $1 \in J$ and $\left|\mathcal{A}^{\prime}\right|=\left|\Lambda\left(\mathcal{A}^{\prime}\right)\right|=\left|\mathcal{S}_{\mathcal{F}, k}((1,1))\right|$, it follows by Proposition 3.6 that $n \in J$ and $\mathcal{A}^{\prime}=\mathcal{S}_{\mathcal{F}, k}((n, 1))$.

Before coming to the proof of Theorem 3.3(ii), we finally determine two nice properties of $\mathcal{F}$-orbits of elements $j \in U(\mathcal{F})$ such that $\mathcal{F}$ is compressed wrt $j$. This will be very useful when dealing with the case $k=2$ of Theorem 3.3(ii).

Proposition 3.8 Let $\mathcal{F}$ and $J$ be as in Theorem 3.3. Let $j^{*} \in J$.

(i) If $\mathrm{O}_{\mathcal{F}}\left(j^{*}\right) \backslash\left\{j^{*}\right\} \neq \phi$ then $\mathcal{F}\left(j^{*}\right)=\mathcal{F}$ and $\mathrm{O}_{\mathcal{F}}\left(j^{*}\right)=J$.

(ii) If $\mathrm{O}_{\mathcal{F}}\left(j^{*}\right)=\left\{j^{*}\right\}$ then $\mathrm{O}_{\mathcal{F}}(j)=\{j\}$ for all $j \in J$.

Proof. Suppose $\mathrm{O}_{\mathcal{F}}\left(j^{*}\right) \backslash\left\{j^{*}\right\} \neq \phi$. Suppose $\mathcal{F}\left(j^{*}\right) \neq \mathcal{F}$. Let $F \in \mathcal{F} \backslash \mathcal{F}\left(j^{*}\right)$ and $j^{\prime} \in$ $\mathrm{O}_{\mathcal{F}}\left(j^{*}\right) \backslash\left\{j^{*}\right\}$. So $j^{\prime} \notin F$ since $F \notin \mathcal{F}\left(j^{*}\right)=\mathcal{F}\left(j^{\prime}\right)$. But then, since $\mathcal{F}$ is compressed wrt $j^{*}$, for any $f \in F$ we have $(F \backslash\{f\}) \cup\left\{j^{*}\right\} \in \mathcal{F}\left(j^{*}\right) \backslash \mathcal{F}\left(j^{\prime}\right)$, which contradicts $\mathcal{F}\left(j^{*}\right)=\mathcal{F}\left(j^{\prime}\right)$. So $\mathcal{F}\left(j^{*}\right)=\mathcal{F}$.

Let $j \in J$. Suppose $j \notin \mathrm{O}_{\mathcal{F}}\left(j^{*}\right)$. So $\mathcal{F}(j) \subsetneq \mathcal{F}\left(j^{*}\right)$ as $\mathcal{F}\left(j^{*}\right)=\mathcal{F}$. Let $F^{*} \in$ $\mathcal{F}\left(j^{*}\right) \backslash \mathcal{F}(j)$. Since $\mathcal{F}$ is compressed wrt $j$, we have $\left(F^{*} \backslash\left\{j^{*}\right\}\right) \cup\{j\} \in \mathcal{F} \backslash \mathcal{F}\left(j^{*}\right)$, which contradicts $\mathcal{F}\left(j^{*}\right)=\mathcal{F}$. Therefore $J \subseteq \mathrm{O}_{\mathcal{F}}\left(j^{*}\right)$. Also, $\mathrm{O}_{\mathcal{F}}\left(j^{*}\right) \subseteq J$ because if $j \in \mathrm{O}_{\mathcal{F}}\left(j^{*}\right)$ then $\mathcal{F}(j)=\mathcal{F}\left(j^{*}\right)=\mathcal{F}$. Hence $(\mathrm{i})$.

Now suppose $\mathrm{O}_{\mathcal{F}}\left(j^{*}\right)=\left\{j^{*}\right\}$ and $\mathrm{O}_{\mathcal{F}}(j) \backslash\{j\} \neq \phi$ for some $j \in J$. By $(\mathrm{i}), \mathrm{O}_{\mathcal{F}}(j)=$ $J$. So $j^{*} \in \mathrm{O}_{\mathcal{F}}(j)$, and hence, by definition, $\mathrm{O}_{\mathcal{F}}\left(j^{*}\right)=\mathrm{O}_{\mathcal{F}}(j)$; but this contradicts $\mathrm{O}_{\mathcal{F}}(j) \backslash\{j\} \neq \phi=\mathrm{O}_{\mathcal{F}}\left(j^{*}\right) \backslash\left\{j^{*}\right\}$. Hence (ii).

Proof of Theorem 3.3(ii). By Proposition 3.6, $\left|\mathcal{S}_{\mathcal{F}, k}((j, b))\right|=\left|\mathcal{S}_{\mathcal{F}, k}((1,1))\right|$ iff $(j, b) \in$ $J \times[k]$. Also, if $k=2$ and $\mathcal{A}^{*}=\left\{F \in \mathcal{F}: F \cap\left(\mathrm{O}_{\mathcal{F}}(1) \times[2]\right) \in \mathcal{X}\right\}$ for some $\mathcal{X} \in \operatorname{ex}\left(\mathcal{S}_{\mathrm{O}_{\mathcal{F}}(1), 2}\right)$ then $\left|\mathcal{A}^{*}\right|=\left|\mathcal{S}_{\mathcal{F}, 2}((1,1))\right|$ as $\mathcal{S}_{\mathcal{F}, 2}((1,1))=\left\{F \in \mathcal{F}: F \cap\left(\mathrm{O}_{\mathcal{F}}(1) \times[2]\right) \in \mathcal{X}^{*}\right\}$, where $\mathcal{X}^{*}=\mathcal{S}_{\mathrm{O}_{\mathcal{F}}(1), 2}((1,1)) \in \operatorname{ex}\left(\mathcal{S}_{\mathrm{O}_{\mathcal{F}}(1), 2}\right)$. By Theorem 3.3(i), the sufficiency condition follows. 
We now continue on the Proof of Theorem 3.3(i) to prove the necessary condition. Therefore, we now consider equality in (6). This gives us equality in (4) together with $\left|\mathcal{A}_{0}\right|=\left|\mathcal{S}_{\mathcal{F}_{0}, k}((1,1))\right|$ and $\left|\mathcal{A}_{i}\langle(n, i)\rangle\right|=\left|\mathcal{S}_{\mathcal{F}_{1}, k}((1,1))\right|, i=1, \ldots, k$.

Since we are proving the result by induction on $n$, we may assume that $\mathcal{F}(n) \neq \phi$ and $n \in \gamma\left(A^{*}\right)$ for some $A^{*} \in \mathcal{A}^{*}$. If $|U(\mathcal{F}(n))|=1$ then $\gamma\left(A^{*}\right)=\{n\}$ and $\mathcal{A}^{*}=\left\{A^{*}\right\}$. So we assume that $|U(\mathcal{F}(n))| \geq 2$, which implies $|U(\mathcal{F}\langle n\rangle)| \geq 1$. Thus, for each $i \in[k]$, we have $\left|\mathcal{A}_{i}\langle(n, i)\rangle\right|=\left|\mathcal{S}_{\mathcal{F}_{1}, k}((1,1))\right|>0$.

Let $J_{0}:=\left\{j_{0} \in[n-1]: \mathcal{F}_{0}\right.$ is compressed wrt $\left.j_{0}\right\}$ and $J_{1}:=\left\{j_{1} \in[n-1]: \mathcal{F}_{1}\right.$ is compressed wrt $\left.j_{1}\right\}$. Clearly, $1 \in J_{0} \cap J_{1}$.

Consider first $k \geq 3$. Since we have equality in (4), it follows that $\mathcal{B}=\phi$ and $\mathcal{A}_{i}=\mathcal{A}((n, i)), i=1, \ldots, k$. Thus, by (3) and (5), $\mathcal{A}^{-}:=\mathcal{A}_{0} \cup \bigcup_{i=1}^{k} \mathcal{A}\langle(n, i)\rangle$ is an intersecting sub-family of $\mathcal{S}_{\mathcal{F}_{0} \cup \mathcal{F}_{1}, k}$. Now, by the inductive hypothesis and $(5), \mathcal{A}_{1}\langle(n, 1)\rangle=$ $\mathcal{S}_{\mathcal{F}_{1}, k}\left(\left(j_{1}, 1\right)\right)$ for some $j_{1} \in J_{1}$. Since $\mathcal{A}((n, 1))=\mathcal{A}_{1}$, we have $\mathcal{A}\langle(n, 1)\rangle=\mathcal{S}_{\mathcal{F}_{1}, k}\left(\left(j_{1}, 1\right)\right)$, and hence $\mathcal{A}^{-} \subseteq \mathcal{S}_{\mathcal{F}_{0} \cup \mathcal{F}_{1}, k}\left(\left(j_{1}, 1\right)\right)$ by Lemma 3.4. So $\mathcal{A} \subseteq \mathcal{S}_{\mathcal{F}, k}\left(\left(j_{1}, 1\right)\right)$. Since we have equality in (6), Proposition 3.6 gives us $j_{1} \in J$ and $\mathcal{A}=\mathcal{S}_{\mathcal{F}, k}\left(\left(j_{1}, 1\right)\right.$. Therefore, by Lemmas 3.5 and $3.7, \mathcal{A}^{*}$ is as required.

Next, consider $k=2$ and $\left|\mathrm{O}_{\mathcal{F}}(1)\right|>1$. By Proposition 3.8(i), $\mathcal{F}=\mathcal{F}(1)$. By Corollary 2.2(i), $\mathcal{A}^{*}=\left\{F \in \mathcal{F}: F \cap\left(\mathrm{O}_{\mathcal{F}}(1) \times[2]\right) \in \mathcal{X}\right\}$ for some $\mathcal{X} \in \operatorname{ex}\left(\mathcal{S}_{\mathrm{O}_{\mathcal{F}}(1), 2}\right)$.

Finally, consider $k=2$ and $\mathrm{O}_{\mathcal{F}}(1)=\{1\}$. Suppose $\mathcal{F}_{0}=\phi$. Then $\mathcal{F}=\mathcal{F}(n)$. If $\mathcal{F}(n) \backslash \mathcal{F}(1) \neq \phi$ and $F \in \mathcal{F}(n) \backslash \mathcal{F}(1)$ then, given that $1 \in J$, we have $(F \backslash\{n\}) \cup\{1\} \in \mathcal{F}_{0}$, a contradiction. So $\mathcal{F}(n) \backslash \mathcal{F}(1)=\phi$, and hence, since $\mathcal{F}(n)=\mathcal{F}, \mathcal{F}(n)=\mathcal{F}(1)$; but this contradicts $\mathrm{O}_{\mathcal{F}}(1)=\{1\}$. So $\mathcal{F}_{0} \neq \phi$, and hence $\left|\mathcal{A}_{0}\right|>0$ as $\left|\mathcal{A}_{0}\right|=\left|\mathcal{S}_{\mathcal{F}_{0}, 2}((1,1))\right|$ and $1 \in J_{0}$. It remains to consider the following three cases.

Case 1: $\left|\mathrm{O}_{\mathcal{F}_{0}}(1)\right|=1$. By the inductive hypothesis and $(3), \mathcal{A}_{0}=\mathcal{S}_{\mathcal{F}_{0}, 2}\left(\left(j_{0}, 1\right)\right)$ for some $j_{0} \in J_{0}$. Clearly, $\mathcal{A}_{0} \cup \mathcal{A}_{1}$ and $\mathcal{A}_{0} \cup \mathcal{A}_{2}$ are intersecting. By Lemma 3.4, we therefore have $\mathcal{A}_{0} \cup \mathcal{A}_{1}, \mathcal{A}_{0} \cup \mathcal{A}_{2} \subseteq \mathcal{S}_{\mathcal{F}, 2}\left(\left(j_{0}, 1\right)\right)$, and hence $\mathcal{A} \subseteq \mathcal{S}_{\mathcal{F}, 2}\left(\left(j_{0}, 1\right)\right)$. Since we have equality in (6), Proposition 3.6 gives us $j_{0} \in J$ and $\mathcal{A}=\mathcal{S}_{\mathcal{F}, k}\left(\left(j_{0}, 1\right)\right.$. By Lemma 3.7, $\mathcal{A}^{\prime}=\mathcal{S}_{\mathcal{F}, k}((j, 1))$, where $j \in\left\{j_{0}, n\right\} \cap J$. Since $1 \in J$ and $\mathrm{O}_{\mathcal{F}}(1)=\{1\}$, Proposition 3.8(ii) gives us $\mathrm{O}_{\mathcal{F}}(j)=\{j\}$. Since $\Gamma\left(\mathcal{A}^{*}\right)=\Gamma\left(\mathcal{A}^{\prime}\right)=\mathcal{F}(j)$, it follows by Corollary 2.2(ii) that $\mathcal{A}^{*}=\mathcal{S}_{\mathcal{F}, k}(j, b)$ for some $b \in[2]$.

Case 2: $\left|\mathrm{O}_{\mathcal{F}_{1}}(1)\right|=1$. By the inductive hypothesis and $(5), \mathcal{A}_{1}\langle(n, 1)\rangle=\mathcal{S}_{\mathcal{F}_{1}, 2}\left(\left(j_{1}, 1\right)\right)$ for some $j_{1} \in J_{1}$. Since $\mathcal{A}_{0} \cup \mathcal{A}_{1}\langle(n, 1)\rangle$ is an intersecting sub-family of $\mathcal{S}_{\mathcal{F}_{0} \cup \mathcal{F}_{1}, k}$, Lemma 3.4 gives us $\mathcal{A}_{0} \cup \mathcal{A}_{1}\langle(n, 1)\rangle \subseteq \mathcal{S}_{\mathcal{F}_{0} \cup \mathcal{F}_{1}, 2}\left(\left(j_{1}, 1\right)\right)$. So $\mathcal{A}_{0} \subseteq \mathcal{S}_{\mathcal{F}_{0}, 2}\left(\left(j_{1}, 1\right)\right)$. Since $\left|\mathcal{A}_{0}\right|=$ $\left|\mathcal{S}_{\mathcal{F}_{0}, 2}((1,1))\right|$, it follows by Proposition 3.6 that $j_{1} \in J_{0}$ and $\mathcal{A}_{0}=\mathcal{S}_{\mathcal{F}_{0}, 2}\left(\left(j_{1}, 1\right)\right)$. As in Case 1, this leads us to the desired result.

Case 3: $\left|\mathrm{O}_{\mathcal{F}_{0}}(1)\right|>1,\left|\mathrm{O}_{\mathcal{F}_{1}}(1)\right|>1$. By Proposition 3.8(i), $\mathcal{F}_{0}(1)=\mathcal{F}_{0}$ and $\mathcal{F}_{1}(1)=$ $\mathcal{F}_{1}$. So $\mathcal{F}(1)=\mathcal{F}$. Thus, by Corollary $2.2(\mathrm{ii}), \mathcal{A}^{*}=\mathcal{S}_{\mathcal{F}, 2}((1, b))$ for some $b \in[2]$.

Proof of Theorem 1.5. By Theorem 3.3, $(1,1) \in L\left(\mathcal{S}_{\mathcal{F}, k}\right)$ and, moreover, $\mathcal{S}_{\mathcal{F}, k}((1,1)) \in$ $\operatorname{ex}\left(\mathcal{S}_{\mathcal{F}, k}\right)$; hence $\mathcal{S}_{\mathcal{F}, k}$ is EKR. By Theorem 3.3(ii), if $k \geq 3$ then $\mathcal{S}_{\mathcal{F}, k}$ is strictly EKR. Now consider $k=2$. If $\mathcal{S}_{\mathcal{F}, 2}$ is not strictly EKR then, by Theorem 3.3(ii) and Corollary 2.2(ii), $\left|\mathrm{O}_{\mathcal{F}}(1)\right| \geq 3$; the converse holds by Proposition 2.3 with $f=1$. 


\section{Proof of Theorem 1.6}

We now give a simple proof of Theorem 1.2 and prove the stronger Theorem 1.6. Thus, in the following, $\mathcal{F}$ is taken to be $r$-uniform. Our first simple observation is that

$$
f^{*} \in L(\mathcal{F}) \Leftrightarrow\left(f^{*}, 1\right) \in L\left(\mathcal{S}_{\mathcal{F}, k}\right)
$$

since for all $f \in U(\mathcal{F}), k^{r-1}|\mathcal{F}(f)|=k^{r-1}\left|\Gamma\left(\mathcal{S}_{\mathcal{F}, k}((f, 1))\right)\right|=\left|\mathcal{S}_{\mathcal{F}, k}((f, 1))\right|$.

For $\mathcal{A} \subseteq \mathcal{S}_{\mathcal{F}, k}$, let $\Theta^{q}(\mathcal{A}):=\left\{\theta_{k}^{q}(A): A \in \mathcal{A}\right\}$. Suppose $\mathcal{F}=\{F\}$ and $\mathcal{A}$ is intersecting. If $A \in \mathcal{A}$ and $q \in[k-1]$ then $\theta_{k}^{q}(A) \notin \mathcal{A}$ as $\theta_{k}^{q}(A) \cap A=\phi$. So $\mathcal{A}, \Theta^{1}(\mathcal{A}), \ldots, \Theta^{k-1}(\mathcal{A})$ are $k$ disjoint copies of the same intersecting family, and hence $k|\mathcal{A}| \leq\left|\mathcal{S}_{F, k}\right|=k^{r}$. Therefore

$$
\mathcal{A} \subset \mathcal{S}_{F, k} \text { intersecting } \Rightarrow|\mathcal{A}| \leq k^{r-1},
$$

which proves Theorem 1.1(i) since $\left|\mathcal{S}_{F, k}((f, 1))\right|=k^{r-1}$.

Proof of Theorem 1.2. Let $\mathcal{F}$ be EKR, and let $\mathcal{A} \subset \mathcal{S}_{\mathcal{F}, k}$ be intersecting. Clearly, $\Gamma(\mathcal{A})$ is intersecting. Thus, for $f^{*} \in L(\mathcal{F}),|\Gamma(\mathcal{A})| \leq\left|\mathcal{F}\left(f^{*}\right)\right|$. For any $F \in \Gamma(\mathcal{A})$, let $\mathcal{A}_{F}:=\mathcal{A} \cap \mathcal{S}_{F, k}$. By $(9),\left|\mathcal{A}_{F}\right| \leq k^{r-1}$. Thus, for any $b \in[k]$,

$$
|\mathcal{A}|=\sum_{F \in \Gamma(\mathcal{A})}\left|\mathcal{A}_{F}\right| \leq k^{r-1}|\Gamma(\mathcal{A})| \leq k^{r-1}\left|\mathcal{F}\left(f^{*}\right)\right|=\left|\mathcal{S}_{\mathcal{F}, k}\left(\left(f^{*}, b\right)\right)\right|
$$

and hence result.

Proof of Theorem 1.6. We continue on the proof of Theorem 1.2, and we now consider equality in $(10)$. So $\Gamma(\mathcal{A})=\mathcal{F}\left(f^{*}\right)$ and for all $F \in \Gamma(\mathcal{A}),\left|\mathcal{A}_{F}\right|=k^{r-1}$. Thus, by (9),

$$
\text { for all } F \in \Gamma(\mathcal{A}), \mathcal{A}_{F} \in \operatorname{ex}\left(\mathcal{S}_{F, k}\right) \text {. }
$$

Case 1: $k \geq 3$. Let $F^{*} \in \Gamma(\mathcal{A})$. By (11) and Theorem 1.1(ii), $\mathcal{A}_{F}=\mathcal{S}_{F, k}\left(\left(a^{*}, b^{*}\right)\right)$ for some $\left(a^{*}, b^{*}\right) \in F^{*} \times[k]$. By Lemma 3.4, $\mathcal{A} \subseteq \mathcal{S}_{\mathcal{F}, k}\left(\left(a^{*}, b^{*}\right)\right)$. So $\mathcal{S}_{\mathcal{F}, k}$ is strictly EKR.

Case 2: $k=2$ and for all $f \in L(\mathcal{F}),\left|\mathrm{O}_{\mathcal{F}}(f)\right| \leq 2$. Let $X:=\bigcap_{F \in \Gamma(\mathcal{A})} F$. By (11) and Theorem 2.1, $|X| \geq 1$. Let $f^{\prime} \in X$; so $\Gamma(\mathcal{A}) \subseteq \mathcal{F}\left(f^{\prime}\right)$. Since $|\Gamma(\mathcal{A})|=\left|\mathcal{F}\left(f^{*}\right)\right|$ and $f^{*} \in L(\mathcal{F}), \Gamma(\mathcal{A})=\mathcal{F}\left(f^{\prime}\right)$ and $f^{\prime} \in L(\mathcal{F})$. So $\left|\mathrm{O}_{\mathcal{F}}\left(f^{\prime}\right)\right| \leq 2$. Since $\mathcal{A} \in \operatorname{ex}\left(\mathcal{S}_{\mathcal{F}\left(f^{\prime}\right), 2}\right)$ (by (11)), it follows by Corollary 2.2(ii) that $\mathcal{A}$ is a star of $\mathcal{S}_{\mathcal{F}, 2}$. So $\mathcal{S}_{\mathcal{F}, 2}$ is strictly EKR.

Case 3: $k=2$ and $\left|\mathrm{O}_{\mathcal{F}}(f)\right| \geq 3$ for some $f \in L(\mathcal{F})$. By $(8),(f, 1) \in L\left(\mathcal{S}_{\mathcal{F}, 2}\right)$. Thus, by Proposition $2.3, \mathcal{S}_{\mathcal{F}, 2}$ is not strictly EKR.

Acknowledgements. The author is indebted to an anonymous referee for careful checking of the paper and suggestions that led to a significant improvement of the presentation. The author also wishes to thank Fred C. Holroyd for helpful comments. 


\section{References}

[1] C. Berge, Nombres de coloration de l'hypergraphe h-parti complet, in: Hypergraph Seminar (Columbus, Ohio 1972), Lecture Notes in Math., Vol. 411, Springer, Berlin, 1974, 13-20.

[2] B. Bollobás, I. Leader, An Erdôs-Ko-Rado theorem for signed sets, Comput. Math. Appl. 34 (1997) 9-13.

[3] M. Deza, P. Frankl, Erdós-Ko-Rado theorem - 22 years later, SIAM J. Algebraic Discrete Methods 4 (1983) 419-431.

[4] K. Engel, An Erdôs-Ko-Rado theorem for the subcubes of a cube, Combinatorica 4 (1984) 133-140.

[5] P.L. Erdôs, U. Faigle, W. Kern, A group-theoretic setting for some intersecting Sperner families, Combin. Probab. Comput. 1 (1992) 323-334.

[6] P. Erdôs, C. Ko and R. Rado, Intersection theorems for systems of finite sets, Quart. J. Math. Oxford (2) 12 (1961) 313-320.

[7] P. Frankl, The shifting technique in extremal set theory, in: Combinatorial Surveys (C. Whitehead, Ed.), Cambridge Univ. Press, London/New York, 1987, 81-110.

[8] H.-D.O.F. Gronau, More on the Erdôs-Ko-Rado theorem for integer sequences, J. Combin. Theory (A) 35 (1983) 279-288.

[9] F.C. Holroyd and J. Talbot, Graphs with the Erdôs-Ko-Rado property, Discrete Math. 293 (2005) 165-176.

[10] G.O.H. Katona, A simple proof of the Erdôs-Chao Ko-Rado theorem, J. Combin. Theory (B) 12 (1972) 183-184.

[11] M.L. Livingston, An ordered version of the Erdôs-Ko-Rado Theorem, J. Combin. Theory (A) 26 (1979) 162-165.

[12] J.-C. Meyer, Quelques problèmes concernant les cliques des hypergraphes $k$-complets et $q$-parti $h$-complets, in: Hypergraph Seminar (Columbus, Ohio 1972), Lecture Notes in Math., Vol. 411, Springer, Berlin, 1974, 127-139.

[13] A. Moon, An analogue of the Erdôs-Ko-Rado theorem for the Hamming schemes H(n,q), J. Combin. Theory (A) 32 (1982) 386-390. 